\title{
Effect of Afatinib plasma levels and pharmacokinetics-related genetic polymorphisms on the degree of side effects in patients with non-small cell lung cancer
}

\author{
Yoshihisa Fukuda ${ }^{1}$, Hideki Hayashi ${ }^{1,2,3}$, Hirotoshi lihara ${ }^{2,3}$, Naoki Mizusaki $^{2}$, Chiemi Hirose ${ }^{3}$, \\ Komei Yanase ${ }^{4}$, Yasushi Ohno ${ }^{4}$, Yoshinori Itoh ${ }^{3}$, Tadashi Sugiyama ${ }^{1,2}$, Michiko Obara ${ }^{1}$ \\ ${ }^{I}$ Community Healthcare Pharmacy, Gifu Pharmaceutical University, Japan, ${ }^{2}$ Pharmacy Practice and Social Science, \\ Gifu Pharmaceutical University, Japan, ${ }^{3}$ Department of Pharmacy, Gifu University Hospital, Japan, ${ }^{4} 2$ nd Department \\ of Internal Medicine, Gifu University School of Medicine, Japan
}

\section{Background}

Epidermal growth factor receptor-tyrosine kinase inhibitors (EGFR-TKIs) represent the first-line treatment for patients with advanced EGFR mutation-positive non-small-cell lung cancer. Afatinib (AFA) is a second-generation EGFR-TKI having excellent therapeutic effects. However, severe diarrhea and skin disorders are observed at high frequency, and these side effects often lead to treatment interruption because of the low quality of life (QOL) experienced by the patients. The relationship between individual variations and onset of these side effects remains to be elucidated. This study aims at revealing the association between these side effects, pharmacokinetics, and related genetic polymorphisms.

Methods

Patients receiving AFA were recruited at the Gifu University Hospital from July 2014 to June 2017. AFA plasma concentrations were measured at day 9 when the concentrations reached the steady state (early phase) and when the prescription dose was stable for more than 1 month (stable phase). We analyzed SNPs in ABCB1, ABCG2, and FMO3 previously identified as genes related to AFA pharmacokinetics. The side effects were classified in accordance with the Common Terminology Criteria for Adverse Events v.4.0. This study was approved by the ethics committee.

Results

Both the incidence rate of diarrhea and acneiform eruption was more than $80 \%$. Correlation was observed between the AFA plasma concentration and degree of seriousness of diarrhea in the early phase. Pharmacokinetics-related genetic polymorphisms were found to influence the evolution of diarrhea. Particularly, the AFA plasma concentration was higher and diarrhea was more severe in patients carrying the A allele of ABCG2 C421A. No relation between the evolution of side effects and genetic polymorphisms and either diarrhea in the maintenance phase or acneiform eruption in the early and maintenance phases was observed.

Conclusions

Seriousness of diarrhea is influenced by drug plasma concentrations in the early phase, influenced by genetic polymorphisms related to TKI pharmacokinetics. Therefore, this particular genetic polymorphism can be tested before AFA administration and the dose individually adapted to every patient can be controlled, leading to reduction of side effects, QOL improvement, and patient compliance maintenance for therapeutic effects. 